\title{
FUNDAMENTAL LIMITATIONS OF THE DECAY OF GENERALIZED ENERGY IN CONTROLLED (DISCRETE-TIME) NONLINEAR SYSTEMS SUBJECT TO STATE AND INPUT CONSTRAINTS
}

\author{
ISTVÁN SELEK ${ }^{a, *}, \operatorname{ENSO~IKONEN~}^{a}$ \\ ${ }^{a}$ Systems Engineering Research Group \\ University of Oulu, Pentti Kaiteran katu 1, 90570 Oulu, Finland \\ e-mail: \{istvan.selek, enso.ikonen\}@oulu.fi
}

\begin{abstract}
This paper is devoted to the analysis of fundamental limitations regarding closed-loop control performance of discrete-time nonlinear systems subject to hard constraints (which are nonlinear in state and manipulated input variables). The control performance for the problem of interest is quantified by the decline (decay) of the generalized energy of the controlled system. The paper develops (upper and lower) barriers bounding the decay of the system's generalized energy, which can be achieved over a set of asymptotically stabilizing feedback laws. The corresponding problem is treated without the loss of generality, resulting in a theoretical framework that provides a solid basis for practical implementations. To enhance understanding, the main results are illustrated in a simple example.
\end{abstract}

Keywords: decay rate maximization, Lyapunov function, nonlinear control systems.

\section{Introduction}

Since its first appearance, the theoretical framework introduced by Lyapunov became one of the substantial tools for the analysis of control systems and evaluation of control performance. The theory is built around the utilization of positive definite, radially unbounded functions that are defined over the state space of the system of interest. As the core concept, these functions bear the name of the inventor and are regarded as Lyapunov functions. Since the related theory roots in Lagrangian and Hamiltonian mechanics, Lyapunov functions can be interpreted (and considered by many) as the generalized energy of the system of interest. Using these, Lyapunov recognized that the stability and convergence properties of dynamical systems with feedback are analogous to the gradual decline (decay) of the generalized energy.

Consequently, this concept highlights the role of feedback from a different perspective which may be interpreted as follows: the primary role of feedback is to reduce the generalized energy of the system of interest to a global minimum. The decay of the generalized energy implies asymptotic stability of the closed-loop system

*Corresponding author around the desired point in state space (usually around the origin).

For a nonlinear dynamical system, the design of a feedback law is a challenging task, and the controlled system must satisfy many criteria. First and foremost, asymptotic stability must be guaranteed; however, among others, fast convergence is always desired. Utilizing Lyapunov's framework, the convergence characteristics-thus the dynamic behavior of the closed-loop system-can be characterized by the decay rate of the generalized energy. To achieve fast convergence, high decay rate is to be achieved which depends on (a) system characteristics (e.g., state evolution dynamics, constraints), (b) the applied feedback law and (c) properties of the Lyapunov function which defines the generalized energy.

The maximization of the decay rate is a timely research topic and has been receiving much attention from the control community (Prieur et al., 2011; Feyzmahdavian et al., 2013; Aranda-Escolástico et al., 2018; Hu et al., 2003; Buhl and Lohmann, 2009). Here, the central problem of interest is the design of a feedback law for a given dynamical system which yields the "fastest possible" convergence. For unconstrained linear time-invariant (LTI) systems the 
classical results relying on the theory of linear matrix inequalities (LMIs) are summarized by Boyd et al. (1994). However, in the presence of (state and control) constraints and nonlinearities, the related problem involves more challenges (Hu et al., 2003; Buhl and Lohmann, 2009).

In general, the utilization of the Lyapunov framework for control synthesis and analysis of stability receives considerably great attention from the research community. These cover (i) the design of the control law (Al'brekht, 1961; Scokaert and Rawlings, 1998; Bemporad et al., 2000; Grushkovskaya and Zuyev, 2014; Li et al., 2018) and/or (ii) the design of Lyapunov functions (Fu, 1993; Duda, 2012; Kaczorek, 2007; Lenka, 2019) for various systems of interest. Depending on the application, the design phases are executed in series (e.g., given an optimality criterion design the control law) or in synergy (e.g., given the system of interest design the Lyapunov function and a stabilizing control law subject to some performance criteria).

This paper, however, is fundamentally different from most of the research related to the utilization/application of the Lyapunov framework and approaches the problem of interest from a different perspective. Here, the focus is not on the design; that is, we are not interested in constructing feedback laws and/or Lyapunov functions for a system or a family of systems. Instead, our interest is in the analysis of the performance under feedback. With that said, the core problem which is addressed in this paper is the determination of the maximum decay rate of the generalized energy over a set of feedback laws where the dynamical system, the Lyapunov function (i.e., the function which characterizes the generalized energy) as well as the set of feedback laws are given. A theoretical framework is developed for general nonlinear, constrained discrete-time systems to provide bounds for the fastest admissible decay of generalized energy in case feedback is used. The presented approach generalizes the authors' recent work on the topic where linearity (affinity) was assumed, and constrained, LTI systems were considered (Selek and Ikonen, 2018).

The paper is organized as follows: Section 2 provides the preliminaries, gives problem-related definitions and formulates the problem of the fastest possible decay rate of generalized energy for constrained nonlinear discrete-time systems. Section 3 provide a controllability analysis of the system of interest and formulates the main results. Section 4 addresses the computational aspects related to the implementation of the main results, including the computation of the barrier which bound the decay rate of the generalized energy. To enhance understanding, the theoretical results developed in the paper are put into practice in the form of a simple example which is considered in Section 5. Finally, Section 6 provides a brief summary of the contents of the paper and draws the conclusions.
1.1. Notation and preliminaries. The sets of real, nonnegative real and positive real numbers are denoted by $\mathbb{R}, \mathbb{R}_{0}$ and $\mathbb{R}_{>0}$, respectively. The set of integers, set of nonnegative and positive integers are denoted by $\mathbb{Z}, \mathbb{N}_{0}$ and $\mathbb{N}$, respectively. Likewise, for any $K \in \mathbb{N}_{0}$, $\mathbb{N}_{0: K}:=\{0,1, \ldots, K\}$ and $\mathbb{N}_{K}:=\{1, \ldots, K\}$. Given a vector $x \in \mathbb{R}^{n},\|x\|_{2}$ and $\|x\|_{\infty}$ denote its Euclidean and maximum norm, respectively. Given two sets $\mathcal{A}$ and $\mathcal{B}$, $\mathcal{A}=\mathcal{B}$ denotes the equality of sets, that is, if $\mathcal{A} \subseteq \mathcal{B}$ and $\mathcal{B} \subseteq \mathcal{A}$ we write $\mathcal{A}=\mathcal{B}$. Correspondingly, $\mathcal{A} \subsetneq \mathcal{B}$ means that $\mathcal{A}$ is a proper (strict) subset of $\mathcal{B}$, i.e., $\mathcal{A} \subseteq \mathcal{B}$ and $\mathcal{A} \neq \mathcal{B}$. Let $\mathcal{A} \subsetneq \mathbb{R}^{n}$ be a compact set and let

$$
\mathcal{B}(\mathcal{A}):=\left\{x \in \mathcal{A} \mid \nexists r \in \mathbb{R}_{>0}, \mathcal{D}(x, r) \subsetneq \mathcal{A}\right\}
$$

denote its boundary, where

$$
\mathcal{D}(x, r):=\left\{y \in \mathbb{R}^{n} \mid\|x-y\|_{2} \leq r, r \in \mathbb{R}_{0}\right\}
$$

is a closed ball on $\mathbb{R}^{n}$ centered at $x$ with radius $r$.

\section{Problem description}

This section introduces the problem of interest. For the sake of clarity, the problem description was divided into three subsections which introduce the essential components of the problem including (a) the dynamical system of interest, (b) the generalized energy and (c) the set of (stabilizing) feedback laws. Finally, the problem of interest is defined at the end of the section.

2.1. Dynamical system. In this paper we consider discrete time nonlinear systems of the form

$$
x_{k+1}=f\left(x_{k}, u_{k}\right), \quad k=0,1, \ldots,
$$

where $x_{k} \in \mathbb{R}^{n}$ denotes the (finite dimensional) state vector, $u_{k} \in \mathbb{R}^{m}$ is regarded as manipulated input at time $k \in \mathbb{N}_{0}$ and the function $f: \mathbb{R}^{n} \times \mathbb{R}^{m} \rightarrow \mathbb{R}^{n}$ defines the state evolution on the following assumption.

Assumption 1. For zero manipulated input $\left(u_{k}=0\right)$ the recursion (3a) has a fixed point in the origin, that is, $f(0,0)=0$.

The evolution of the system of interest is restricted by $K_{\text {eq }} \in \mathbb{N}$ equality and $K_{\text {ieq }} \in \mathbb{N}$ inequality constraints, so that

$$
\begin{aligned}
& h_{i}\left(x_{k}, u_{k}\right)=0, \quad \forall i \in \mathbb{N}_{K_{\text {eq }}}, \\
& g_{j}\left(x_{k}, u_{k}\right) \leq 0, \quad \forall j \in \mathbb{N}_{K_{\text {ieq }}},
\end{aligned}
$$

$\forall k \in \mathbb{N}_{0}$, where $h_{i}, g_{j}: \mathbb{R}^{n} \times \mathbb{R}^{m} \rightarrow \mathbb{R}$. Defining the feasible set

$$
\begin{aligned}
& \mathcal{M}:=\left\{[x, u] \in \mathbb{R}^{n+m} \mid h_{i}(x, u)=0,\right. \forall i \in \mathbb{N}_{K_{\mathrm{eq}}} \\
&\left.g_{j}(x, u) \leq 0, \quad \forall j \in \mathbb{N}_{K_{\text {ieq }}}\right\},
\end{aligned}
$$

we also make the following assumption: 
Assumption 2. The feasible set $\mathcal{M}$

(i) includes the origin in its interior (i.e., $[0,0] \in \mathcal{M}$ ),

(ii) is compact and simply connected.

Using this, the restrictions posed by the constraint system (3b)-3c can be expressed in a more compact form

$$
\left[x_{k}, u_{k}\right] \in \mathcal{M}, \quad \forall k \in \mathbb{N}_{0}
$$

which will be used throughout this paper to simplify the notation. The orthogonal projection of $\mathcal{M}$ onto the space of states $\mathbb{R}^{n}$ defines

$$
\mathcal{X}:=\left\{x \in \mathbb{R}^{n} \mid \exists u \in \mathbb{R}^{m},[x, u] \in \mathcal{M}\right\},
$$

which will include all state trajectories due to the fact that (4) is a hard constraint. In other words, $\mathcal{X}$ is a positively invariant set of system (3). What is more, it is supposed to meet the following assumption:

Assumption 3. The set $\mathcal{X}$ is measurable on $\mathbb{R}^{n}$.

To be able to analyze the performance of the system of interest in the presence of (closed-loop) control, one must ensure that (3) satisfies specific controllability requirements. The problem addressed in this paper relies on the concept of null-controllability which plays a central role in the sequel and defined as follows:

Definition 1. System (3) is null-controllable on $\mathcal{R} \subseteq \mathbb{R}^{n}$, $(0 \in \mathcal{R})$ if $\forall x_{0} \in \mathcal{R}$, there exists a finite $K \in \mathbb{N}_{0}$ and an input sequence $\left\{u_{0}, \ldots, u_{K}\right\}$ such that $x_{K+1}=0$ subject to (3).

The study of null-controllability of system (3) is a problem of its own and is not addressed by this paper. Instead, the satisfaction of the controllability requirement is ensured by the following assumption:

Assumption 4. System (3) is null-controllable on $\mathcal{X}$.

It defines the fourth pillar of the presented work. However, in comparison with Assumptions 1-3, Assumption 4 might be too restrictive. In such cases, the "relaxation" of the last assumption is possible, so that the results are to be developed using a null-controllable subset of $\mathcal{X}$. Nevertheless, this is going to be omitted for the sake of clarity.

2.2. Generalized energy. Let a function $V: \mathbb{R}^{n} \rightarrow$ $\mathbb{R}_{0}$ be defined over the state space of (3a) satisfying the following properties:

(i) positive definiteness: $V(x)>0, \forall x \in \mathbb{R}^{n} \backslash 0$ and $V(x)=0$ otherwise; (ii) for any $v_{1}, v_{2} \in \mathbb{R}_{0}$ we have $v_{1}<v_{2} \Leftrightarrow \mathcal{V}\left(v_{1}\right) \subsetneq$ $\mathcal{V}\left(v_{2}\right)$, where

$$
\mathcal{V}(v):=\left\{x \in \mathbb{R}^{n} \mid V(x) \leq v\right\}
$$

defines the level set of $V(x)$;

(iii) the level set $\mathcal{V}(v)$ is compact and simply connected $\forall v \in \mathbb{R}_{0}$.

The function $V(x)$ is regarded as generalized energy throughout this paper.

2.3. Set of asymptotically stabilizing feedback laws. By definition, any feedback law applied to (3) which makes the solution $x_{k}=0, \forall k \in \mathbb{N}_{0}$ asymptotically stable in the Lyapunov sense implies that the generalized energy will approach zero and vice versa, that is,

$$
\lim _{k \rightarrow \infty} x_{k}=0 \Leftrightarrow \lim _{k \rightarrow \infty} V\left(x_{k}\right)=0 .
$$

Given this relation, the paper aims to study the fundamental limitations on the gradual decline (decay) of generalized energy which appears under (asymptotically stabilizing) feedback. However, unlike many valuable contributions of the related field, we are not interested in the synthesis of a feedback law or laws which achieve good (best) performance with respect to some criteria quantifying the decay of the generalized energy. Here, we are interested in determining the greatest lower bound on the decay of the generalized energy over a set which includes all stabilizing feedback laws. The related problem is stated at the end of this section, but first, let the set of stabilizing feedback laws be defined as follows: Given $x_{0} \in \mathcal{X}$,

$$
\begin{aligned}
\mathcal{F}\left(x_{0}\right):=\left\{\mu: \mathbb{R}^{n} \times \mathbb{R}_{0} \rightarrow \mathbb{R}^{m} \mid\right. & \lim _{k \rightarrow \infty} V\left(x_{k}\right)=0, \text { s.t. } \\
& u_{k}=\mu\left(x_{k}, k\right) \\
& x_{k+1}=f\left(x_{k}, u_{k}\right) \\
& {\left[x_{k}, u_{k}\right] \in \mathcal{M} } \\
& \left.\forall k \in \mathbb{N}_{0}\right\}
\end{aligned}
$$

where the mapping $\mu$ is regarded as feedback law or control policy.

Remark 1. The set $\mathcal{F}\left(x_{0}\right)$ includes all feedback laws (i.e., linear, nonlinear, optimal in some sense, the ones which utilize full state information as well as the ones which use partial state information through observations, etc.) which steer (3) back to the origin from the initial state $x_{0} \in \mathcal{X}$.

Putting these together, a problem related to the decay of the generalized energy over $\mathcal{F}\left(x_{0}\right)$ is addressed and defined as follows: 
Problem 1. Given an initial state $x_{0} \in \mathcal{X}$, find a sequence $\left\{V_{0}^{\star}, V_{1}^{\star}, \ldots\right\},\left(V_{\tau}^{\star} \in \mathbb{R}_{0}, \forall \tau \in \mathbb{N}_{0}\right)$ so that

$$
V_{\tau}^{\star}=\sup _{v \in \mathbb{R}_{0}} v
$$

subject to

$$
\begin{aligned}
u_{k}-\mu\left(x_{k}, k\right) & =0, \\
x_{k+1}-f\left(x_{k}, u_{k}\right)= & 0, \\
h_{i}\left(x_{k}, u_{k}\right)= & 0, \\
g_{j}\left(x_{k}, u_{k}\right) \leq & 0, \\
v-V\left(x_{\tau}\right) \leq & 0, \\
& \forall i \in \mathbb{N}_{K_{\text {eq }}}, \\
& \forall j \in \mathbb{N}_{K_{\text {ieq }}}, \\
& \forall k \in \mathbb{N}_{0: \tau}, \\
& \forall \mu \in \mathcal{F}\left(x_{0}\right) .
\end{aligned}
$$

The variable $V_{\tau}^{\star}$ defines the greatest lower bound of the generalized energy of the system of interest at time $\tau \in \mathbb{N}_{0}$ which can be achieved by means of feedback. Consequently, for a given initial condition $x_{0} \in \mathcal{X}$ there exists no feedback law $\mu: \mathbb{R}^{n} \rightarrow \mathbb{R}^{m}$ such that $V\left(x_{\tau}\right)<$ $V_{\tau}^{\star}\left(\forall \tau \in \mathbb{N}_{0}\right)$ subject to $(3)$.

Since the computation of $V_{\tau}^{\star}$ requires solution of an optimization problem at each time instant $\tau$ which can be solved independently, the value of $V_{\tau}^{\star}$ is mapped to the particular time instant $\tau$ and unaffected by the choice of the lookahead horizon (e.g., finite or infinite). Using these, the obtained sequence $\left\{V_{0}^{\star}, V_{1}^{\star}, \ldots\right\}$ is referred to as the fastest admissible decay of the generalized energy of the system of interest.

It is essential to point out that a single stabilizing feedback law which "produces" the optimal sequence $\left\{V_{0}^{\star}, V_{1}^{\star}, \ldots\right\}$ might not exist due to the fact that the solution to Problem 1 is obtained over $\mathcal{F}\left(x_{0}\right)$, that is, the components of the optimal sequence can be determined by different feedback laws.

\section{Bounds of the fastest admissible decay}

Similarly to the linear case (Selek and Ikonen, 2018), the solution to the outlined problem is case specific. Its dependence on the initial state $x_{0}$ makes the generalization of the solution highly challenging. However, as demonstrated by Selek and Ikonen (2018) it is possible to derive an approximate solution with no loss of generality, using barriers $\left\{\underline{V}_{0}, \underline{V}_{1}, \ldots\right\}$ and $\left\{\bar{V}_{0}, \bar{V}_{1}, \ldots\right\}$ which bound $\left\{V_{0}^{\star}, V_{1}^{\star}, \ldots\right\}$ such that

$$
\underline{V}_{i} \leq V_{i}^{\star} \leq \bar{V}_{i}, \quad \underline{V}_{i}, \bar{V}_{i} \in \mathbb{R}_{0}, \quad \forall i \in \mathbb{N}_{0} .
$$

It must be pointed out that in practice the barriers are computed $\forall i \in \mathbb{N}$, since obviously $\underline{V}_{0}=\bar{V}_{0}=$ $V\left(x_{0}\right)$ given that the initial state $x_{0}$ is known. The development of the barriers rests on the concept of $i$-step null-controllable sets which eliminate the dependence of the approximate solution on the initial state while introducing some conservatism in return. Informally, the $i$-step null-controllable set $\overline{\mathcal{X}}_{i} \subseteq \mathcal{X}$ or attraction domain is a set of states which can be steered to the origin in finite time using a control sequence which is at most $i$ steps long (Polyak and Shcherbakov, 2009). Precisely, we have the following:

Definition 2. Let $\overline{\mathcal{X}} \subseteq \mathcal{X}$ and $\overline{\mathcal{X}} \backslash 0 \neq \emptyset$. The set $\overline{\mathcal{X}}$ is $i$-step null-controllable if $\forall x_{0} \in \overline{\mathcal{X}}$ there exists a finite $i \in \mathbb{N}$ and an input sequence $\left\{u_{0}, \ldots, u_{i-1}\right\}$ such that $x_{i}=0$ subject to (3).

To be consistent with the definition, we say that $\overline{\mathcal{X}}$ is 0 -step null-controllable if $\overline{\mathcal{X}}=\{0\}$. Consequently, by definition the $i$-step null controllable set for $i=0$ is (Darup and Mönnigmann, 2013),

$$
\overline{\mathcal{X}}_{0}=0
$$

and the ones for $i>0$ are determined by the following (forward) iteration

$$
\begin{array}{r}
\overline{\mathcal{X}}_{i}:=\left\{x \in \mathcal{X} \mid \exists u \in \mathbb{R}^{m}: f(x, u) \in \overline{\mathcal{X}}_{i-1},\right. \\
[x, u] \in \mathcal{M}\} .
\end{array}
$$

The iteration above results in a nested sequence of $\overline{\mathcal{X}}_{i} \subseteq \overline{\mathcal{X}}_{i+1}$ of null controllable sets. The nested structure is a direct consequence of Definition 2. To show this, consider $x \in \overline{\mathcal{X}}_{i}$ with the corresponding control sequence $\left\{u_{0}, \ldots, u_{i-1}\right\}$. Adding a zero to the sequence $\left\{u_{0}, \ldots, u_{i-1}, u_{i}=0\right\}$ implies that $x \in \overline{\mathcal{X}}_{i+1}$ by definition. Furthermore, adding a sequence of $j \in \mathbb{N}$ zeros to $\left\{u_{0}, \ldots, u_{i-1}\right\}$ implies that $x \in \overline{\mathcal{X}}_{i+j}$, thus $x \in \overline{\mathcal{X}}_{i} \Rightarrow \overline{\mathcal{X}}_{i+j}, \forall j \in \mathbb{N}$. Consequently $\overline{\mathcal{X}}_{i} \subseteq \overline{\mathcal{X}}_{i+1}$.

On the other hand, it is less obvious that the $i$-step null-controllable sets must "grow" with $i$ as stated by the following lemma.

Lemma 1. If (3) is null controllable on $\mathcal{X}$ then $\forall i \in \mathbb{N}$, $\overline{\mathcal{X}}_{i-1} \subsetneq \mathcal{X} \Rightarrow \overline{\mathcal{X}}_{i-1} \subsetneq \overline{\mathcal{X}}_{i}$.

Proof. Since the set $\mathcal{M}$ includes the origin, $\overline{\mathcal{X}}_{0}$ can be controlled to itself, that is, if $x_{k} \in \overline{\mathcal{X}}_{0}$ there exists $u_{k}$ (more precisely $u_{k}=0$ ) such that $x_{k+1} \in \overline{\mathcal{X}}_{0}$ subject to (3). Consequently, (10) implies that $\overline{\mathcal{X}}_{0} \subseteq \overline{\mathcal{X}}_{1}$. On the other hand, $\overline{\mathcal{X}}_{1} \neq \overline{\mathcal{X}}_{0}$ since (3) is null-controllable on $\mathcal{X}$; thus, for $i=1, \overline{\mathcal{X}}_{0} \subsetneq \overline{\mathcal{X}}_{1}$. Assume that $\overline{\mathcal{X}}_{i-1} \subsetneq \mathcal{X}$ and $\overline{\mathcal{X}}_{i-1} \subsetneq \overline{\mathcal{X}}_{i}$ hold for every $i \in \mathbb{N}_{j}$. Then, from (10) it follows that $\overline{\mathcal{X}}_{j} \subseteq \overline{\mathcal{X}}_{j+1}$. If $\overline{\mathcal{X}}_{j} \subsetneq \mathcal{X}$ then (similarly to the case of $i=1) \overline{\overline{\mathcal{X}}}_{j+1} \neq \overline{\mathcal{X}}_{j}$ since (3) is null-controllable on $\mathcal{X}$; thus $\overline{\mathcal{X}}_{j} \subsetneq \overline{\mathcal{X}}_{j+1}$. Otherwise, from (10) it follows that $\overline{\mathcal{X}}_{j} \subseteq \mathcal{X}, \forall j \in \mathbb{N}_{0}$; thus $\overline{\mathcal{X}}_{j}=\mathcal{X}$ and $\overline{\mathcal{X}}_{j} \subseteq \overline{\mathcal{X}}_{j+1}$ implies that $\overline{\mathcal{X}}_{j+1}=\overline{\mathcal{X}}_{j}=\mathcal{X}$. By induction the outlined statements complete the proof. 

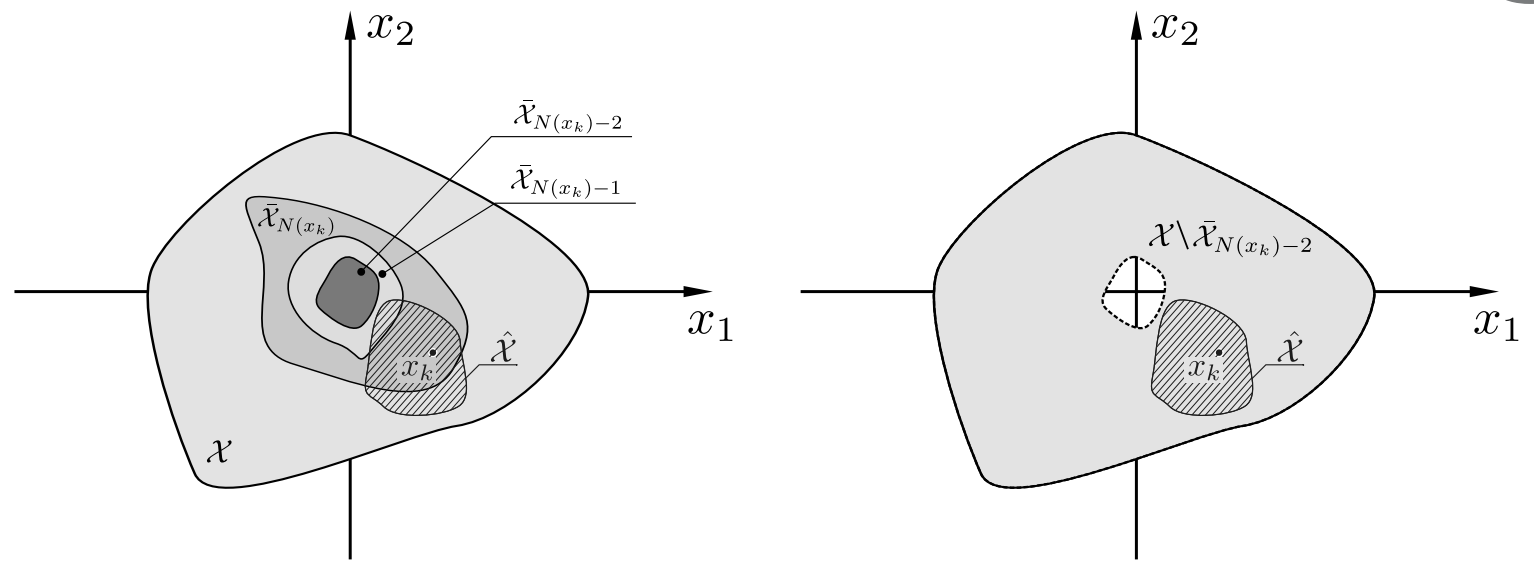

Fig. 1. Left: an example demonstrating the arrangement (nested sequence) of the null-controllable sets $\left(\overline{\mathcal{X}}_{N\left(x_{k}\right)}, \overline{\mathcal{X}}_{N\left(x_{k}\right)-1}\right.$, $\overline{\mathcal{X}}_{N\left(x_{k}\right)-2}$ ) on the positively invariant set $\mathcal{X}$. The state $x_{k}$ is marked by the dot and the set of states which are reachable from $x_{k}$ in one step $\hat{\mathcal{X}}$ is hatched. Right: an example demonstrating the one-step ahead state transition. As pointed out by Lemma 2, $\hat{\mathcal{X}} \subseteq \mathcal{X} \backslash \overline{\mathcal{X}}_{N\left(x_{k}\right)-2}$, which is colored in gray. Note that, in this particular case, $\mathcal{X} \backslash \overline{\mathcal{X}}_{N\left(x_{k}\right)-2}$ is non-simply connected. If the set includes its limit points, the boundary is highlighted by a continuous line; otherwise, a dashed line is used to mark the boundary. The interiors are colored in both the cases.

Since the positively invariant set $\mathcal{X}$ defined by the constraint system represents the "largest" feasible set on the state space and the $i$-step null-controllable sets cannot grow infinitively. This is a direct consequence of the proof of Lemma 1 formally expressed as the following result.

Corollary 1. If (3) is null controllable on $\mathcal{X}$ then $\forall i \in \mathbb{N}$, $\overline{\mathcal{X}}_{i-1}=\mathcal{X} \Rightarrow \overline{\mathcal{X}}_{i}=\mathcal{X}$.

Proof. See the proof of Lemma1.

Utilizing the outlined properties of the sequence of $i$-step null-controllable sets, the minimum number of steps required to reduce the generalized energy of the system of interest to zero for any $x \in \mathcal{X}$ can be obtained by solving the following optimization problem:

$$
N(x)=\min \left\{i \mid x \in \overline{\mathcal{X}}_{i}, i \in \mathbb{N}_{0}\right\},
$$

which makes $x \in \mathcal{X} N(x)$-step null-controllable. Using this, an important property concerning state transition is to be highlighted by the following result:

Lemma 2. For all $N\left(x_{k}\right) \geq 2, x_{k} \in \overline{\mathcal{X}}_{N\left(x_{k}\right)} \Rightarrow x_{k+1} \in$ $\mathcal{X} \backslash \overline{\mathcal{X}}_{N\left(x_{k}\right)-2}$ subject to 3 .

Proof. From (10) it follows that the state $x_{k} \in \overline{\mathcal{X}}_{N\left(x_{k}\right)}$ cannot be steered to $\overline{\mathcal{X}}_{N\left(x_{k}\right)-2}$ in one step since (11) implies $x_{k} \notin \overline{\mathcal{X}}_{N\left(x_{k}\right)-1}$. In other words, if $x_{k} \in \overline{\mathcal{X}}_{N\left(x_{k}\right)}$ there exists no $u_{k} \in \mathbb{R}^{m}$ such that $x_{k+1} \in \overline{\mathcal{X}}_{N\left(x_{k}\right)-2}$ subject to (3). Since $\mathcal{X}$ is a positively invariant set of (3), $x_{k+1} \in \mathcal{X}$; consequently, $x_{k+1} \in \mathcal{X} \backslash \overline{\mathcal{X}}_{N\left(x_{k}\right)-2}$.

A visual interpretation of Lemma2 2 is given by Fig. 1 considering a two-dimensional state space. Lemma 2 is used to construct one-step-ahead bounds for the decay of the generalized energy as stated by the following result.
Theorem 1. For all $x_{k} \in \mathcal{X} \backslash \overline{\mathcal{X}}_{1}$,

$$
\begin{aligned}
& \max \left\{V(x) \mid x \in \overline{\mathcal{X}}_{N\left(x_{k}\right)-1}\right\} \\
& \geq \min \left\{V\left(x_{k+1}\right) \mid \text { s.t. (3) }\right\} \\
& \geq \inf \quad\left\{V(x) \mid x \in \mathcal{X} \backslash \overline{\mathcal{X}}_{N\left(x_{k}\right)-2}\right\} .
\end{aligned}
$$

Proof. From (11) it follows that $x_{k} \in \overline{\mathcal{X}}_{N\left(x_{k}\right)}$. Let $\hat{\mathcal{X}}$ denote the set of states which can be reached from $x_{k}$ in one step subject to (3), that is,

$$
\begin{array}{r}
\hat{\mathcal{X}}:=\left\{x \in \mathcal{X} \mid \exists u \in \mathbb{R}^{m}, x=f\left(x_{k}, u\right),\right. \\
\left.\left[x_{k}, u\right] \in \mathcal{M},\right\}
\end{array}
$$

where Assumption 4, (10) and Lemma1 assure that $\hat{\mathcal{X}} \neq$ $\emptyset$ and $\overline{\mathcal{X}}_{N\left(x_{k}\right)-1} \cap \hat{\mathcal{X}} \neq \emptyset$. Consequently,

$$
\begin{aligned}
& \max \left\{V(x) \mid x \in \overline{\mathcal{X}}_{N\left(x_{k}\right)-1}\right\} \\
& \geq \max \left\{V(x) \mid x \in \overline{\mathcal{X}}_{N\left(x_{k}\right)-1} \cap \hat{\mathcal{X}}\right\} \\
& \geq \min \{V(x) \mid x \in \hat{\mathcal{X}}\}
\end{aligned}
$$

since $\overline{\mathcal{X}}_{N\left(x_{k}\right)-1} \cap \hat{\mathcal{X}} \subseteq \overline{\mathcal{X}}_{N\left(x_{k}\right)-1}$ and $\overline{\mathcal{X}}_{N\left(x_{k}\right)-1} \cap \hat{\mathcal{X}} \subseteq \hat{\mathcal{X}}$.

Similarly, Lemma2 2 assures that $\hat{\mathcal{X}} \subseteq \mathcal{X} \backslash \overline{\mathcal{X}}_{N\left(x_{k}\right)-2}$ and (11) imply that $\overline{\mathcal{X}}_{N\left(x_{k}\right)-2} \neq \mathcal{X}$, which gives

$$
\begin{aligned}
& \min \{V(x) \mid x \in \hat{\mathcal{X}}\} \\
& \geq \inf \left\{V(x) \mid x \in \mathcal{X} \backslash \overline{\mathcal{X}}_{N\left(x_{k}\right)-2}\right\}
\end{aligned}
$$


Remark 2. Accordingly, it is obvious that, for all $x_{k} \in$ $\overline{\mathcal{X}}_{1}$,

$$
\min \left\{V\left(x_{k+1}\right) \mid \text { subject to (3) }\right\}=0,
$$

due to the fact that the origin can be reached from $\overline{\mathcal{X}}_{1}$ in one step subject to (3).

The outlined theorem developed bounds for the one-step-ahead decay of the generalized energy. However, since Theorem 1 utilized the topological structure of the $i$-step null-controllable sets, the same idea can be used to construct the bounding barriers for an arbitrarily long lookahead horizon, simply by considering feedback laws which "enforce" state transition from $\overline{\mathcal{X}}_{k}$ to $\overline{\mathcal{X}}_{k-1}$ at each time step $k$.

The definition of the set of feedback laws (8) implies that $\mathcal{F}\left(x_{0}\right)$ includes control policies which steer the system's state back to the origin in minimum time. It follows from (10) that a feedback law is minimum time if, and only if, the sequence of states generated by the feedback law satisfies $\left\{x_{0}, x_{1}, \ldots, x_{N}\right\} \in$ $\left\{\overline{\mathcal{X}}_{N\left(x_{0}\right)}, \overline{\mathcal{X}}_{N\left(x_{0}\right)-1}, \ldots, \overline{\mathcal{X}}_{0}\right\}$, that is, at each time instant $k, x_{k} \in \overline{\mathcal{X}}_{N\left(x_{0}\right)-k} \Rightarrow x_{k+1} \in \overline{\mathcal{X}}_{N\left(x_{0}\right)-(k+1)}$ subject to (3). Fortunately, it is easy to construct feedback laws which satisfy the outlined criteria. As an example, consider the feedback law of the form

$$
u_{k}=\operatorname{RND}\left(\hat{\mathcal{U}}_{k}\left(x_{k}\right)\right)
$$

where $\operatorname{RND}\left(\hat{\mathcal{U}}_{k}\left(x_{k}\right)\right)$ denotes a randomly chosen element of $\hat{\mathcal{U}}_{k}\left(x_{k}\right)$ and

$$
\begin{gathered}
\hat{\mathcal{U}}_{k}\left(x_{k}\right):=\left\{u \in \mathbb{R}^{m} \mid f\left(x_{k}, u\right) \in \overline{\mathcal{X}}_{N\left(x_{0}\right)-(k+1)},\right. \\
\left.\left[x_{k}, u\right] \in \mathcal{M},\right\} .
\end{gathered}
$$

With no doubt, (17) is minimum time and satisfies (8) thus included in $\mathcal{F}\left(x_{0}\right)$. Putting these together, let us introduce the following notation: for any $i \in \mathbb{Z}$ such that $\overline{\mathcal{X}}_{i} \neq \mathcal{X}$ let

$$
\bar{V}_{i}:= \begin{cases}\max \left\{V(x) \mid x \in \overline{\mathcal{X}}_{i}\right\} & \text { if } i \geq 0 \\ 0 & \text { otherwise }\end{cases}
$$

and

$$
\underline{V}_{i}:= \begin{cases}\inf \left\{V(x) \mid x \in \mathcal{X} \backslash \overline{\mathcal{X}}_{i}\right\} & \text { if } i \geq 0, \\ 0 & \text { otherwise }\end{cases}
$$

Thus given an initial state $x_{0} \in \mathcal{X}$, the barriers bounding the fastest admissible decay of the generalized energy of (3) over $\mathcal{F}\left(x_{0}\right)$ are defined by the following result:
Corollary 2. Given $x_{0} \in \mathcal{X}$ and the corresponding set of feedback laws $\mathcal{F}\left(x_{0}\right)$, we have

$$
\underline{V}_{N\left(x_{0}\right)-(k+2)} \leq V_{k+1}^{\star} \leq \bar{V}_{N\left(x_{0}\right)-(k+1)},
$$

$\forall k \in \mathbb{N}_{0}$ subject to (9).

Proof. Since $\mathcal{F}\left(x_{0}\right)$ includes minimum time feedback laws, the inequality (20) is a direct consequence of Theorem 1 together with Remark 2. See proof of Theorem 1

The derivation of (20) was based on the observation that the set of feedback laws include minimal time controllers. Knowing this it is natural to formulate the question of what if the optimization defined by (9) is executed on a subset of $\mathcal{F}\left(x_{0}\right)$ which is defined, for example, so that the minimum time control laws are removed from $\mathcal{F}\left(x_{0}\right)$. In other words, in what degree (20) is affected in case the original problem (Problem 1) is defined over an arbitrary subset of $\mathcal{F}\left(x_{0}\right)$. The corresponding problem is defined as follows.

Problem 2. Given an initial state $x_{0} \in \mathcal{X}$, find a sequence $\left\{V_{0}^{\star}, V_{1}^{\star}, \ldots\right\},\left(V_{\tau}^{\star} \in \mathbb{R}_{0}, \forall \tau \in \mathbb{N}_{0}\right)$ so that

$$
V_{\tau}^{\star}=\sup _{v \in \mathbb{R}_{0}} v
$$

subject to

$$
\begin{aligned}
u_{k}-\mu\left(x_{k}, k\right)= & 0, \\
x_{k+1}-f\left(x_{k}, u_{k}\right)= & 0, \\
h_{i}\left(x_{k}, u_{k}\right)= & 0, \\
g_{j}\left(x_{k}, u_{k}\right) \leq & 0, \\
v-V\left(x_{\tau}\right) \leq & 0, \\
& \forall i \in \mathbb{N}_{K_{\text {eq }}}, \\
& \forall j \in \mathbb{N}_{K_{\text {ieq }}}, \\
& \forall k \in \mathbb{N}_{0: \tau}, \\
& \forall \mu \in \hat{\mathcal{F}}\left(x_{0}\right) \subseteq \mathcal{F}\left(x_{0}\right) .
\end{aligned}
$$

First and foremost, it is obvious that in case the subset $\hat{\mathcal{F}}\left(x_{0}\right) \subseteq \mathcal{F}\left(x_{0}\right)$ includes at least one minimum time feedback law, 20) is unaffected. In the opposite case, the answer to the formulated question lies in equations (13), (14) and (15). Considering a one-step lookahead, in the absence of minimum time feedback laws there exists a set of time instances $\left\{k_{1}, \ldots, k_{i}\right\}$ for which state transitions from the set $\overline{\mathcal{X}}_{N\left(x_{k_{i}}\right)}$ to $\overline{\mathcal{X}}_{N\left(x_{k_{i}}\right)-1}$ do not occur. For such cases from (13) and (14) it is obvious that $\overline{\mathcal{X}}_{N\left(x_{k}\right)-1} \cap \hat{\mathcal{X}}=\emptyset$. Thus (14) becomes meaningless implying that the upper bound becomes invalid. On the other hand, the lower bound is valid for any cases since the condition $\hat{\mathcal{X}} \subseteq \mathcal{X} \backslash \overline{\mathcal{X}}_{N\left(x_{k}\right)-2}$ holds under any circumstances. This conclusion holds for lookahead horizons of arbitrary size as well. 
The obtained results can be interpreted as follows: it is possible to choose feedback laws that reduce the generalized energy of the system slower than the upper barrier. On the contrary, there exists no admissible feedback law (i.e., $\mu \in \mathcal{F}\left(x_{0}\right)$ ) which reduces the system's generalized energy faster than the lower barrier as stated by the following result.

Theorem 2. Given $x_{0} \in \mathcal{X}$ and an arbitrary set of feedback laws $\hat{\mathcal{F}}\left(x_{0}\right) \subseteq \mathcal{F}\left(x_{0}\right)$, we have

$$
\underline{V}_{N\left(x_{0}\right)-(k+2)} \leq V_{k+1}^{\star},
$$

$\forall k \in \mathbb{N}_{0}$ subject to 21 .

Proof. Inequality (22) is a direct consequence of Theorem 1 See the proof of Theorem 1

The sequence $\quad\left\{\underline{V}_{N\left(x_{0}\right)-2}, \underline{V}_{N\left(x_{0}\right)-3}, \ldots,\right\}$ provided by (22) is going to be referred to as the greatest feasible lower bound of the system's generalized energy $V\left(x_{k+1}\right)$ at any time instant $k \in \mathbb{N}$. In this context, the term feasible lower bound reflects the condition that there are cases when the system's generalized energy gets infinitely close to the barrier at a particular time instant (or time instants) although in other cases the lower barrier might be conservative. However, there are no cases such that the system's generalized energy crosses (lower than) the barrier.

\section{Computational aspects}

This section is devoted to the analysis of the optimization problem which defines the lower barrier. Formula (19) requires the solution of the following optimization problem:

$$
\inf \left\{V(x) \mid x \in \mathcal{X} \backslash \overline{\mathcal{X}}_{i}\right\}
$$

Unlike simple cases where the problem above can be solved analytically, general high dimensional systems require numerical approaches to render a solution to (23). Here, among others the nonconvexity of the optimization domain and/or the objective function might be an issue; however, the main challenge is introduced by the fact that $\mathcal{X} \backslash \overline{\mathcal{X}}_{i}$ might not be simply connected. For example, Fig. 11(right) presents a typical case in a two-dimensional state space where the optimization domain is not simply connected (the domain is highlighted by gray color).

The topological properties of the optimization domain (e.g., simply connected or not) depends on the "arrangement" of the $i$-step null-controllable sets $\overline{\mathcal{X}}_{i}$ versus the positively invariant set $\mathcal{X}$. Regarding this, two cases are distinguished: (a) sets $\mathcal{X}$ and $\overline{\mathcal{X}}_{i}$ share a common boundary and (b) sets $\mathcal{X}$ and $\overline{\mathcal{X}}_{i}$ do not share a common boundary. To assign mathematical content to these cases, the following definition is formulated:
Definition 3. Let $\mathcal{Y} \subsetneq \mathcal{Z}$ be two compact and simply connected sets which are measurable on $\mathbb{R}^{n}$. The sets $\mathcal{Y}$ and $\mathcal{Z}$ share a common boundary if

$$
\forall x \in \mathcal{B}(\mathcal{Y}) \nexists r \in \mathbb{R}_{>0}: \mathcal{D}(x, r) \subsetneq \mathcal{Z}
$$

Correspondingly, the sets $\mathcal{Y}$ and $\mathcal{Z}$ do not share a common boundary if

$$
\forall x \in \mathcal{B}(\mathcal{Y}) \exists r \in \mathbb{R}_{>0}: \mathcal{D}(x, r) \subsetneq \mathcal{Z} .
$$

The graphical interpretation of the outlined cases can be seen in Fig. 2. The reason to distinguish the outlined two cases roots in the fact that unlike (24), property (25) allows a change in the optimization domain and the reformulation of the related optimization problem. More precisely, (25) implies that (23) can be defined as a maximization problem over a simply connected set (replacing the minimization problem over a non-simply connected one).

The related lemma which states the reformulation rests on the following property of the energy function.

Remark 3. By the definition of $V$, for any $v_{1}<v_{2}$ $\left(v_{1}, v_{2} \in \mathbb{R}_{0}\right)$ we have

$$
\forall x \in \mathcal{B}\left(\mathcal{V}\left(v_{1}\right)\right) \exists r \in \mathbb{R}_{>0}: \mathcal{D}(x, r) \subsetneq \mathcal{V}\left(v_{2}\right) .
$$

To show this, assume that $\exists x \in \mathcal{B}\left(\mathcal{V}\left(v_{1}\right)\right)$ for which $\nexists r \in \mathbb{R}_{>0}$ such that $\mathcal{D}(x, r) \subsetneq \mathcal{V}\left(v_{2}\right)$. Definition 1 and $\mathcal{V}\left(v_{1}\right) \subsetneq \mathcal{V}\left(v_{2}\right)$ imply that $x \in \mathcal{B}\left(\mathcal{V}\left(v_{2}\right)\right)$. Since $x \in$ $\mathcal{B}\left(\mathcal{V}\left(v_{1}\right)\right) \Rightarrow V(x)=v_{1}$ and $x \in \mathcal{B}\left(\mathcal{V}\left(v_{2}\right)\right) \Rightarrow V(x)=$ $v_{2}$, we get $v_{1}=v_{2}$ which is a contradiction.

Using this, the equivalent optimization problem is defined as follows.

Lemma 3. If $\overline{\mathcal{X}}_{i} \neq \mathcal{X}$ and $\forall x \in \mathcal{B}\left(\overline{\mathcal{X}}_{i}\right) \exists r \in \mathbb{R}_{>0}$ : $\mathcal{D}(x, r) \subsetneq \mathcal{X}$ then

$$
\begin{aligned}
\inf \left\{V(x) \mid x \in \mathcal{X} \backslash \overline{\mathcal{X}}_{i}\right\} \\
\\
=\max \left\{v \in \mathbb{R}_{0} \mid \mathcal{V}(v) \subseteq \overline{\mathcal{X}}_{i}\right\}
\end{aligned}
$$

Proof. If $\forall x \in \mathcal{B}\left(\overline{\mathcal{X}}_{i}\right) \exists r \in \mathbb{R}_{>0}$ such that $\mathcal{D}(x, r) \subsetneq \mathcal{X}$, it follows from (10) that $\overline{\mathcal{X}}_{i}$ are compact and include the origin in their interior. This implies that $\forall i \in \mathbb{N}_{0} \exists v \in \mathbb{R}_{0}$ such that $\mathcal{V}(v) \subsetneq \overline{\mathcal{X}}_{i}$.

Let $v^{\star}:=\max \left\{v \in \mathbb{R}_{0} \mid \mathcal{V}(v) \subseteq \overline{\mathcal{X}}_{i}\right\}$ and $V^{\star}=$ $\min \left\{V(x) \mid x \in \mathcal{B}\left(\overline{\mathcal{X}}_{i}\right)\right\}$. Since $\mathcal{B}\left(\overline{\mathcal{X}}_{i}\right) \subseteq \overline{\mathcal{X}}_{i}$ it follows from the definitions of $v^{\star}, V^{\star}$ and Remark 3 that $V^{\star} \leq$ $v^{\star}$.

Let us assume that $V^{\star}<v^{\star}$ which implies that $x^{\star}=\arg \min \left\{V(x) \mid x \in \mathcal{B}\left(\overline{\mathcal{X}}_{i}\right)\right\} \in \mathcal{V}\left(v^{\star}\right)$. From Remark 3 it follows that $\exists r \in \mathbb{R}_{>0}$ such that 

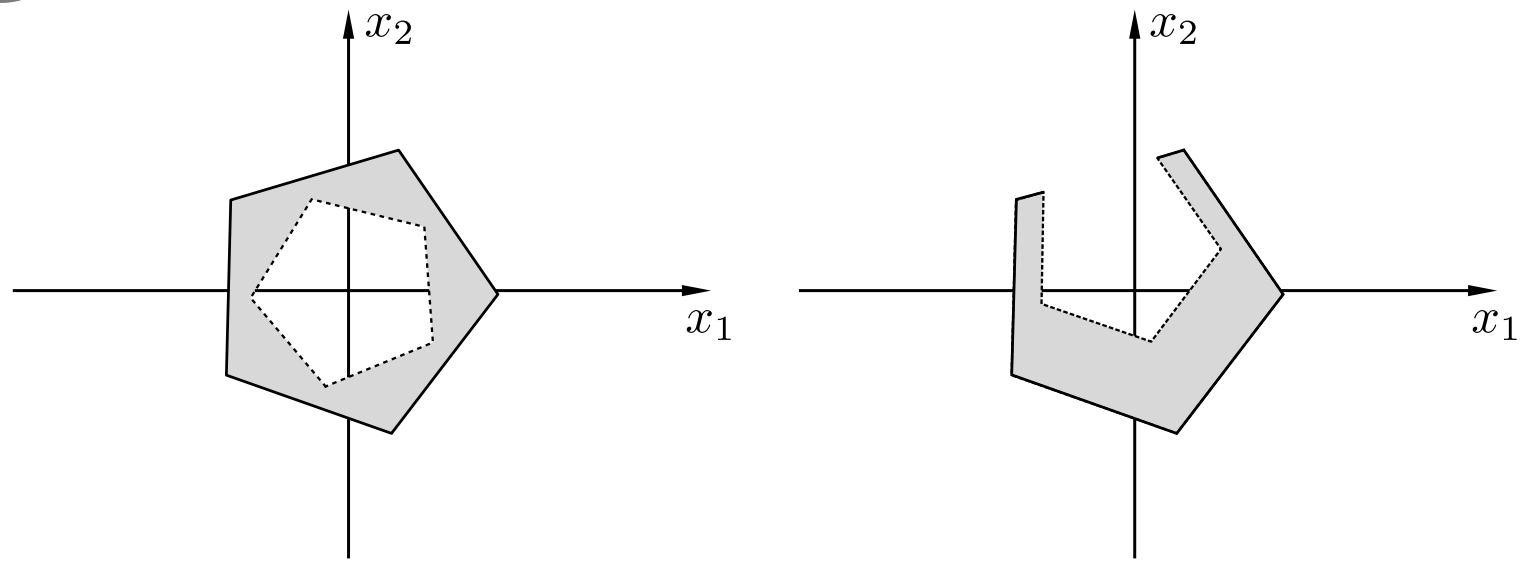

Fig. 2. Example on the left: the difference of two compact sets that do not share a common boundary. The resulting set is non-simply connected. Example on the right: the difference of two compact sets of sets that share a common boundary. In this particular case, the resulted set is simply connected but non-convex. In case the set includes its limit points, the boundary is highlighted by a continuous line; otherwise, the dashed line is used to mark the boundary. The interiors are colored in both cases.

$\mathcal{D}\left(x^{\star}, r\right) \subsetneq \mathcal{V}\left(v^{\star}\right)$ and, consequently, $\mathcal{D}\left(x^{\star}, r\right) \subsetneq \overline{\mathcal{X}}_{i}$ since $\mathcal{V}\left(v^{\star}\right) \subseteq \overline{\mathcal{X}}_{i}$. On the contrary, $\nexists r \in \mathbb{R}_{>0}$ such that $\mathcal{D}\left(\bar{x}^{\star}, r\right) \subsetneq \overline{\mathcal{X}}_{i}$ since $x^{\star} \in \mathcal{B}\left(\overline{\mathcal{X}}_{i}\right)$. Consequently, $\min \left\{V(x) \mid x \in \mathcal{B}\left(\overline{\mathcal{X}}_{i}\right)\right\}=\max \{v \in$ $\left.\mathbb{R} \mid \mathcal{V}(v) \subseteq \overline{\mathcal{X}}_{i}\right\}$ and since $\inf \left\{V(x) \mid x \in \mathcal{X} \backslash \overline{\mathcal{X}}_{i}\right\}=$ $\min \left\{V(x) \mid x \in \mathcal{B}\left(\overline{\mathcal{X}}_{i}\right) \cup \mathcal{X} \backslash \overline{\mathcal{X}}_{i}\right\}$ the proof is completed.

The reformulation of the optimization problem enables the execution of the optimization over a simply connected set. Thus it provides some benefits from the perspective of practical implementation. On the other hand, in practice, the maximization problem might still involve substantial challenges.

\section{Example system}

For the sake of clarity, here, a simple first-order discrete-time integrator is considered as an example. The

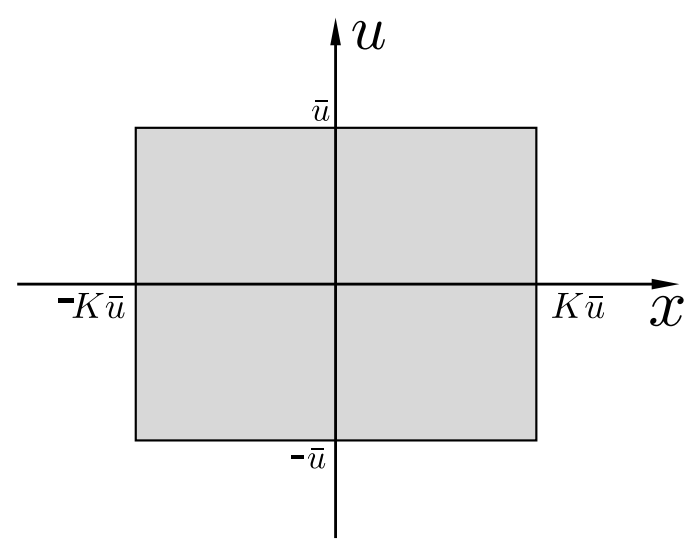

Fig. 3. "Box" constraint (feasible set $\mathcal{M}$ ) of system (27). dynamics of the system are described by

$$
x_{k+1}=x_{k}+u_{k}
$$

subject to the following constraints:

$$
\begin{aligned}
& \left\|x_{k}\right\|_{2} \leq K \bar{u}, \\
& \left\|u_{k}\right\|_{2} \leq \bar{u}, \\
& x_{k}, u_{k} \in \mathbb{R},
\end{aligned}
$$

where $\bar{u} \in \mathbb{R}_{>0}$ and $K \geq 3, K \in \mathbb{N}$. System (27b) defines a "box" on $\mathbb{R}^{2}$ which is depicted in Fig. 3 .

The generalized energy of the system is defined by the quadratic expression $V(x)=x^{2}$ and the initial condition $x_{0}$ is parameterized by a positive constant $\delta \in$ $[0, \bar{u}]$ such that $x_{0}(\delta)=(K-1) \bar{u}+\delta$.

To obtain the barriers which bound the fastest admissible decay of the generalized energy of the outlined system, first the $i$-step null-controllable sets must be characterized. Using the definition determined by (10) in this simple case, these can be derived analytically resulting in the following expression:

$$
\overline{\mathcal{X}}_{i}=\left\{x \in \mathbb{R} \mid\|x\|_{2} \leq i \bar{u}\right\}, \quad \forall i \in \mathbb{N}_{0: K} .
$$

Figure 4 depicts the arrangement of the $i$-step null-controllable sets (bottom) and the generalized energy (top) of the system over the feasible region of the state space.

To highlight the arrangement (pyramid shape) of the null-controllable sets, these are drawn underneath the state space as $i$ grows. Consequently, the $i$-th null controllable set on the state space is obtained by applying orthogonal projection onto the axis parameterized by the variable $x$. By applying projection, the boundaries of the null-controllable sets (i.e., $x=i \bar{u}$ ) uniformly 


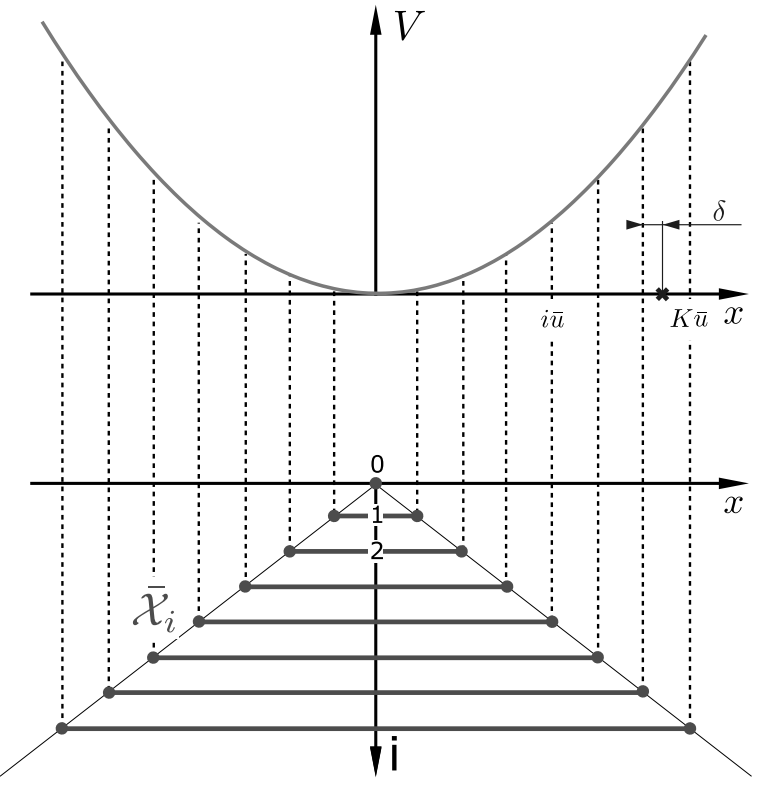

Fig. 4. Energy function (up) and the arrangement of $i$-step null controllable sets (down) of the discrete time integrator (27a). The initial state $x_{0}(\delta)$ is highlighted by the cross.

discretize the state space over the feasible region with a discretization increment of $\bar{u}$.

Using this, (18) and (19) can be evaluated on the generated grid resulting in

$$
\underline{V}_{i}=\bar{V}_{i}= \begin{cases}(i \bar{u})^{2} & \text { if } 0<i<K, \\ 0 & \text { otherwise }\end{cases}
$$

$\forall i \in \mathbb{Z}, i<K$. Here, the equality $\underline{V}_{i}=\bar{V}_{i}$ is unique, which is implied by the unidimensionality of the state space. From a mathematical perspective, the equality is a direct consequence of Lemma3 in one dimension. Given $x_{0}(\delta)$ and applying $(20)$, we get

$$
\begin{aligned}
(K-(k+2))^{2} \bar{u}^{2} & \leq V\left(x_{k+1}\right) \\
& \leq(K-(k+1))^{2} \bar{u}^{2}
\end{aligned}
$$

for all $k \in \mathbb{N}$. The obtained result is easy to verify since given $x_{k}$ the largest possible step towards the origin is $\bar{u}$. For example, consider a minimum-time feedback law of the form

$$
u_{k}= \begin{cases}-x_{k} & \text { if } x_{k} \in \overline{\mathcal{X}}_{1}, \\ -\frac{\left|x_{k}\right|}{x_{k}} \bar{u} & \text { otherwise. }\end{cases}
$$

which enforces the maximum step towards the origin at each time instant. Applying the outlined feedback law to (27a) with initial condition $x_{0}(\delta)$, the state at time instant $k \in \mathbb{N}$ is determined by the following equality:

$$
x_{k}= \begin{cases}0 & \text { if } x_{k-1} \in \overline{\mathcal{X}}_{1}, \\ (K-1) \bar{u}+\delta-k \bar{u} & \text { otherwise. }\end{cases}
$$

Putting these together, we get

$$
\begin{aligned}
& \lim _{\delta \rightarrow 0} V\left(x_{k+1}\right)-\underline{V}_{K-(k+2)}=0, \forall k \in \mathbb{N}, \\
& \lim _{\delta \rightarrow \bar{u}} V\left(x_{k+1}\right)-\bar{V}_{K-(k+1)}=0, \forall k \in \mathbb{N} .
\end{aligned}
$$

Using Fig. 4 the obtained result can be easily verified. Starting from the initial state (denoted by the cross) (31), always applies the maximum possible step towards the origin. Thus, the energy of the system at any time instant gets infinitely close to the lower barrier as parameter $\delta$ approaches zero. Since the feedback law considered in the example is minimum time, the upper barrier is valid as well and approached as parameter $\delta$ tends to $\bar{u}$.

To have a clear view on the validity of the upper barrier, consider the subset $\hat{\mathcal{F}}\left(x_{0}\right)$ of $\mathcal{F}\left(x_{0}\right)$ including only one feedback law

$$
u_{k}= \begin{cases}-x_{k} & \text { if } x_{k} \in \overline{\mathcal{X}}_{1}, \\ -\frac{1}{P} \frac{\left|x_{k}\right|}{x_{k}} \bar{u} & \text { otherwise, }\end{cases}
$$

where $P \in \mathbb{N}, P>2$ is a large positive integer. Similarly to 31 , the outlined law forms a stable system with (27a and reduces the generalized energy to zero. The decay rate, however, is much slower than it was inthe first case due to the step scaling parameter $P$. For simplicity, assume that $\delta=\bar{u}$, which implies that $x_{0}=K \bar{u}$. Applying (34) to (27a), the state at time instant $k \in \mathbb{N}$ is determined by the following equality:

$$
x_{k}= \begin{cases}0 & \text { if } x_{k-1} \in \overline{\mathcal{X}}_{1}, \\ K \bar{u}-\frac{k}{P} \bar{u} & \text { otherwise. }\end{cases}
$$

Using this, it is easy to verify that

$$
\bar{V}_{K-(k+1)}<V\left(x_{k+1}\right)
$$

for $P \geq 2$. As outlined in the previous section, here, the upper barrier is invalid (i.e., it does not bound the fastest admissible decay of generalized energy from above) since $\hat{\mathcal{F}}\left(x_{0}\right)$ does not include a minimum-time feedback law. Consequently, the conservativeness of the lower barrier becomes prominent as $P \rightarrow \infty$,

$$
\begin{aligned}
\lim _{P \rightarrow \infty} \min \left(V\left(x_{k+1}\right)-\underline{V}_{K-(k+2)}\right) & \\
= & \left(K^{2}-(K-2)^{2}\right) \bar{u}^{2}
\end{aligned}
$$

and, similarly,

$$
\lim _{P \rightarrow \infty} \max \left(V\left(x_{k+1}\right)-\underline{V}_{K-(k+2)}\right)=(K \bar{u})^{2} .
$$




\section{Summary and conclusions}

This paper addressed the convergence properties of nonlinear systems under the effect of feedback subject to state and input constraints. The convergence of closed-loop systems over a set of feedback laws was characterized by the decay of the system's generalized energy. Without the loss of generality, barriers were developed which bound the fastest admissible decay of generalized energy. It was shown that the upper barrier is valid under the condition that the set of feedback laws includes minimum-time control policies while the lower barrier is universal, that is, there is no admissible feedback law which reduces the system's energy faster than the outlined bound. The generality of the results was achieved by using controllability and reachability sets which depend (only) on system characteristics (i.e., state evolution dynamics and constraints).

It must be emphasized that the presented results are solely theoretical. Their implementation for case studies might involve substantial challenges. This is induced by the fact that the required calculations (e.g., null-controllable sets, optimization problems) might lead to computationally intractable problems, depending on the features (dimensionality, a specific type of nonlinearity, constraints, etc.) of the underlying problem. On the other hand, the developed results provide a solid basis for practical considerations.

\section{References}

Al'brekht, E.G. (1961). On the optimal stabilization of nonlinear systems, Journal of Applied Mathematics and Mechanics 25(5): 1254-1266.

Aranda-Escolástico, E., Salt, J., Guinaldo, M., Chacón, J. and Dormido, S. (2018). Optimal control for aperiodic dual-rate systems with time-varying delays, Sensors 18(5): 1-19.

Bemporad, A., Torrisit, F.D. and Morarit, M. (2000). Performance analysis of piecewise linear systems and model predictive control systems, IEEE Conference on Decision and Control, Sydney, NSW, Australia, pp. 4957-4962.

Boyd, S., El-Ghaoui, L., Feron, E. and Balakrishnan, V. (1994). Linear Matrix Inequalities in System and Control Theory, SIAM, Philadelphia, PA.

Buhl, M. and Lohmann, B. (2009). Control with exponentially decaying Lyapunov functions and its use for systems with input saturation, European Control Conference, Budapest, Hungary, pp. 3148-3153.

Darup, M.S. and Mönnigmann, M. (2013). Null-controllable set computation for a class of constrained bilinear systems, European Control Conference, Zürich, Switzerland, pp. 2758-2763.
Duda, J. (2012). A Lyapunov functional for a system with a time-varying delay, International Journal of Applied Mathematics and Computer Science 22(2): 327-337, DOI: 10.2478/v10006-012-0024-7.

Feyzmahdavian, H. R., Charalambous, T. and Johansson, M. (2013). On the rate of convergence of continuous-time linear positive systems with heterogeneous time-varying delays, European Control Conference, Zürich, Switzerland, pp. 3372-3377.

$\mathrm{Fu}$, J. (1993). Families of Lyapunov functions for nonlinear systems in critical cases, IEEE Transactions on Automatic Control 38(1): 3-16.

Grushkovskaya, V. and Zuyev, A. (2014). Optimal stabilization problem with minimax cost in a critical case, IEEE Transactions on Automatic Control 59(9): 2512-2517.

Hu, T., Lin, Z. and Shamash, Y. (2003). On maximizing the convergence rate for linear systems with input saturation, IEEE Transactions on Automatic Control 48(7): 1249-1253.

Kaczorek, T. (2007). The choice of the forms of Lyapunov functions for a positive 2D Roesser model, International Journal of Applied Mathematics and Computer Science 17(4): 471-475, DOI: 10.2478/v10006-007-0039-7.

Lenka, B.K. (2019). Time-varying Lyapunov functions and Lyapunov stability of nonautonomous fractional order systems, International Journal of Applied Mathematics 32(1): 111-130.

Li, W., Huang, C. and Zhai, G. (2018). Quadratic performance analysis of switched affine time-varying systems, International Journal of Applied Mathematics and Computer Science 28(3): 429-440, DOI: 10.2478/amcs-2018-0032.

Polyak, B. and Shcherbakov, P. (2009). Ellipsoidal approximations to attraction domains of linear systems with bounded control, Proceedings of the American Control Conference, St. Louis, MO, USA, pp. 5363-5367.

Prieur, C., Tarbouriech, S. and Zaccarian, L. (2011). Improving the performance of linear systems by adding a hybrid loop, 18th IFAC World Congress, Milan, Italy, pp. 6301-6306.

Scokaert, P. and Rawlings, J.B. (1998). Constrained linear quadratic regulation, IEEE Transactions on Automatic Control 43(8): 1163-1169.

Selek, I. and Ikonen, E. (2018). On the bounds of the fastest admissible decay of generalized energy in controlled LTI systems subject to state and input constraints, 15th International Conference on Electrical Engineering, Computing Science and Automatic Control (CCE2018), Mexico City, Mexico, p. ID: 19. 


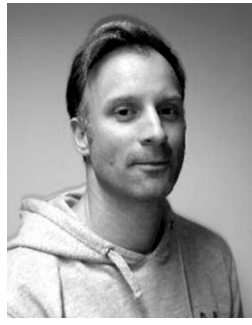

István Selek received his MSc degree in applied mechanics (mechanical engineering) from the Budapest University of Technology and Economics in 2003 and the $\mathrm{PhD}$ degree in systems engineering from the University of Oulu in 2009. $\mathrm{He}$ is currently a postdoctoral researcher of the Systems Engineering Research Group there. His research interests include control theory, methods of mathematical programming and their application to dynamic energy networks.

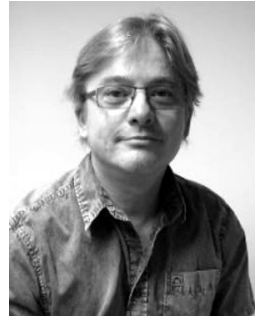

Enso Ikonen is a full professor and the head of the Systems Engineering Research Group of the University of Oulu, Finland. His professorship covers theory, methods and applications of control and systems engineering, with applications in energy systems, in particular. $\mathrm{He}$ is the author of three books and more than 30 journal articles. His research interests are in methods and applications of process control, including modelbased process control, state estimation and computational intelligence in control.

Received: 30 November 2018

Revised: 30 April 2019

Accepted: 25 July 2019 